Emojis: Insights, Affordances and Possibilities for Psychological Science

\author{
Linda K. Kaye ${ }^{1 *}$, Stephanie A. Malone ${ }^{2} \&$ Helen J. Wall ${ }^{1}$ \\ ${ }^{1}$ Edge Hill University, UK; ${ }^{2}$ Australian Catholic University, AUS
}

*Correspondence: Linda.kaye@edgehill.ac.uk (Linda K. Kaye)

We live in a digital society that provides a range of opportunities for virtual interaction. Consequently, emojis have become popular for clarifying online communication. This presents an exciting opportunity for psychologists as these prolific online behaviours can be used to help reveal something unique about contemporary human behaviour.

Keywords: Emojis; online communication; online data; cyberpsychology 


\section{Emojis: Insights, Affordances and Possibilities for Psychological Science}

Communication is central to our lives and draws upon both verbal (e.g., speech) and non-verbal behaviours (e.g., facial expressions) to convey information. Since the rise in Social Networking Sites (SNSs), an exponential growth in online communication has been seen, particularly in text-based forms of interaction (e.g., email, messages, blogs). This can be an effective tool for communicating verbal messages, yet lacks the subtle non-verbal behaviours associated with face-to-face interaction. As a result, compensatory mechanisms have quickly developed to counter the diminished non-verbal channels [1]. One such development is the use of digital behaviours such as emoticons and emojis. Emoticons developed as short character strings, such as :), to portray facial expression [2], with the first reported use occurring in discussion forums in the 1980 s where a smiley face :-) was included to indicate the message was a joke [3]. In the late 1990s, a Japanese company transformed these basic representations into colourful pictorial images for use with mobile phones. These images were termed "emojis". They are now popular worldwide and used across numerous digital platforms and contexts [4]. Indeed, these symbols have become a frequently used expression of emotion and communicative intent, with up to $92 \%$ of the online population using them [5]. Within this, these portray a wide breadth of emotions; far-beyond those of simple smiles and frowns (e.g., sarcasm, surprise, crying).

Although research into the use and interpretation of emoticons/emojis is still in its infancy, it has already been shown that they disambiguate the communicative intent behind messages [6], serve important verbal and non-verbal functions in communication [7], and can even provide insight into the user's personality [8]. Given the global phenomenon of emoticons and emojis, numerous studies have highlighted the ease with which large data sets can be obtained from people's use of emojis. That is, the accessible nature of many online platforms (e.g., Twitter, blogs) provides psychologists with a wealth of everyday contemporary communicative behaviours, occurring in meaningful, real-world contexts. How can psychologists make the 
most of these rich, available data? By making effective use of emojis in research, our understanding of real-world communication can be enhanced outside of the typical laboratory setting, thereby sidestepping certain limitations of the experimental method, such as experimenter priming or issues with random participant assignment [9].

The pervasive use of digital environments, such as SNSs, offer new opportunities to examine human behaviour and communication through a contemporary lens (e.g., in the environments in which an increasing proportion of our communication behaviours are actually taking place). This novel methodological focus can arguably develop the current understanding of human behaviour, communication and perception, while also promoting new lines of enquiry. As such, this is an area ripe for scientific exploration and discovery. Already, some recent research has begun this work, providing preliminary insights into the role of emoji and emoticons in modern communication.

\section{Functions of communication}

Until now, much research on communicated emotion has primarily been examined in the context of face-to-face or spoken verbal interaction, specifically when understanding interpersonal, dynamic functions. However, it is imperative to consider whether the underlying factors and processes associated with well-researched face-to-face emotional displays, operate in equivalent ways within the contemporary online portrayal of emotion via emoji/emoticon.

Drawing upon the method of corpus linguistics [10], the bountiful occurrence of emojis in real-world online text provides a new means to examine the function of contemporary interactional communication and emotion portrayal. Pioneering work on cognitive analysis of emoticons highlights that they can serve as useful forms of nonverbal behaviour in addition to revealing new insights into cognitive and neural mechanisms involved in digital communication. For example, previous research has explored the neuro-correlates of emoticon- 
enriched sentences to identify which brain regions are engaged when sentences included both verbal and nonverbal (i.e., emoticon) cues [7]. Interestingly, findings showed activation of both the left and the right inferior frontal gyri (the latter of these being an area associated with inhibitory control), suggesting differential representation to that of typical left-hemispherical predominance in verbal tasks.

Other research exploring emoji usage has found they serve both intrapersonal and interpersonal functions for the user and interaction partner(s) [6]. Specifically, from an interpersonal perspective, users are highly aware of how emojis can reduce discourse ambiguity (see Box 1), yet also intrapersonally acknowledge they can aid expression through establishing emotional tone that is often lost with the absence of face-to-face interaction, thus providing the user with a toolkit for clarifying emotional concepts. Regardless of these benefits, users are highly contextually-aware and tend to alter the frequency of their emoji usage depending upon the specific communicative platform (e.g., usage is greater in social-networking than email) [6]. This brings new insights into the psychological conceptualisations of emotional expression and communication. Specifically, virtual communications may be more "considered" and consciously controlled than in traditional face-to-face expressions, which have been found to be more spontaneous in nature [11]. This therefore reveals that there may be unique underpinnings associated with emotional communication via emoji usage which may be inherently different from face-to-face expression. This is indicative of how our virtual interactions may impact upon our emotional and social affordances, perceptions, and appraisals.

\section{Personality impressions}


In addition to examining the use of emoticons and emojis to portray emotions, online data can also be utilised to investigate how personality is communicated online and whether virtual portrayals of emotion impact how the user is viewed by others. Recent research has investigated the formation of online impressions of personality [8]. The findings indicate that naïve raters are able to judge an individual's extraversion and openness to experience with reasonable levels of accuracy (i.e. self-other agreement), on the basis of viewing his or her Facebook profile [8]. In contrast, openness to experience is largely undetectable in face-to-face first impressions [12]. Taken together, this illustrates that there is clearly something unique about aspects of virtual behaviours, which distinguish them from our traditional conceptualisations of human behaviour.

\section{Moving forward}

As online interaction is so prevalent in daily life, it is important to gain an enhanced understanding of the underlying factors relating to this form of interaction. Digital data provides a novel and exciting means by which to re-examine many psychological concepts relating to perception and communication, including emotional expression, emotional mimicry, emotional appraisal, pragmatics, and intention-detection. By examining the commonalities between the arguably parallel forms of face-to-face versus online behaviours, it is possible to establish whether contemporary behaviours such as emojis can be considered true forms of emotion on a neurological and interpersonal level.

Taken together, we encourage researchers to consider the utility of online behaviours to help reveal novel mechanisms that can develop our understanding of human behaviour. The utility of emojis is one of many examples that may be instrumental here (see Box 2 for another example). Importantly, this movement pertains to a more general need for adopting insights 
from the realm of cyberpsychology if we are to conceptualise human psychology within contemporary, digital society and make use of all the insights that it might offer into human nature.

[Box 1 with Figure 1 about here]

Figure 1. An example of the communicative power of emojis in reducing ambiguity in written discourse

[Box 2 with Figure $2^{1}$ about here]

Figure 2. An example of an emoji Likert scale

\section{References}

1. Walther, J. B. and D'Addario, K. P. (2001) The impacts of emoticons on message interpretation in computer-mediated communication. Soc Sci Comput Rev, 19, $323 \mathrm{e} 345$

2. Coburn, K. L. (2009). Instant messenger use by individuals with Asperger's syndrome. Unpublished dissertation. (University of Pittsburgh, Pittsburgh).

3. Dresner, E. and Herring, S. C. (2010) Functions of the nonverbal in CMC: Emoticons and illocutionary force. Comm Theor, 20 (3), 249-268

\footnotetext{
${ }^{1}$ The emojis used in Figure 2 are provided free by Yellowicon and can be downloaded from IconEasy www.iconeasy.com/inconset/emotion-orange-icons. For further validation details, see Moore, Steiner and Conlon (2013).
} 
4. Novak, P. K. et al. (2015) Sentiment of emojis. PloS One, 10(12), e0144296

5. Emogi Consumer Science Team (2015) 2015 Emoji Report. Retrieved October 14, 2016 from http://emogi.com/documents/Emoji_Report_2015.pdf

6. Kaye, L. K. et al. (2016) "Turn that frown upside-down": A contextual account of emoticon usage on different virtual platforms. Comput Hum Behav, 60, 463-467

7. Yuasa, M. et al. (2011) Brain activity when reading sentences and emoticons: An fMRI study of verbal and nonverbal communication. Electronics and Communications in Japan, 94(5), 17-24.

8. Wall, H. J. et al. (2016) An exploration of psychological factors on emoticon usage and implications for judgement accuracy. Comput Hum Behav, 62, 70-78

9. Bless, H. and Burger, A. M. (2016) A closer look at Social Psychologists' Silver Bullet: Inevitable and Evitable side effects of the experimental approach. Perspect Psychol Sci, $11(2), 296-308$

10. McEnery, T. and Hardie, A. (2012) Corpus linguistics: Method, theory and practise. Cambridge: Cambridge University Press

11. Buck, R. (1994) Social and emotional functions in facial expression and communication: the readout hypothesis. Biol Psychol, 38 (2e3), 95e115

12. Funder, D. C. (1999) Personality judgment: A realistic approach to person perception. London: Academic Press 


\section{Box 1. Communicative power of emojis}

Emoji can clarify the intended tone of a message. In Figure 1, the inclusion of the "wink" emoji adds information to the written discourse. This particular exchange is based on the scenario that Matthew enjoys running, but he is not a very fast runner. At school, Matthew and his friends entered a running race in which Matthew came last in the race, and finds out that his friend, Julia, came first.

Without the emoji, the message would be interpreted simply that Matthew was close to winning the race. However, Julia's use of a wink emoji suggests she is being sarcastic in her message. This is just one example of how emojis add non-verbal information to verbal discourse in a somewhat equivalent way to gestures or facial expressions during spoken speech.

\section{Box 2. Emojis as potential survey instruments}

With the prolific use of emojis, shared understandings of their meanings are emerging. Specifically, for basic emotions (e.g., happiness, sadness), individuals share awareness of the emotion they are representing. This presents possible uses for these icons. For example, rather than using traditional rating scales, we could move towards utilising emojis rather than the more subjective linguistic increments on a Likert scale. This would indeed be particularly relevant for research with children or those who have difficulty accessing and interpreting language. An example of how this may be represented is provided in Figure 2. 